\title{
Robust DEA under discrete uncertain data: a case study of Iranian electricity distribution companies
}

\author{
Ashkan Hafezalkotob • Elham Haji-Sami • \\ Hashem Omrani
}

Received: 30 January 2014/ Accepted: 30 June 2014/Published online: 18 December 2014

(c) The Author(s) 2014. This article is published with open access at Springerlink.com

\begin{abstract}
Crisp input and output data are fundamentally indispensable in traditional data envelopment analysis (DEA). However, the real-world problems often deal with imprecise or ambiguous data. In this paper, we propose a novel robust data envelopment model (RDEA) to investigate the efficiencies of decision-making units (DMU) when there are discrete uncertain input and output data. The method is based upon the discrete robust optimization approaches proposed by Mulvey et al. (1995) that utilizes probable scenarios to capture the effect of ambiguous data in the case study. Our primary concern in this research is evaluating electricity distribution companies under uncertainty about input/output data. To illustrate the ability of proposed model, a numerical example of 38 Iranian electricity distribution companies is investigated. There are a large amount ambiguous data about these companies. Some electricity distribution companies may not report clear and real statistics to the government. Thus, it is needed to utilize a prominent approach to deal with this uncertainty. The results reveal that the RDEA model is suitable and reliable for target setting based on decision makers (DM's) preferences when there are uncertain input/output data.
\end{abstract}

\footnotetext{
A. Hafezalkotob $(\bowtie) \cdot$ E. Haji-Sami

Industrial Engineering College, Islamic Azad University, South

Tehran Branch, Entezari Alley, Oskoui Alley, Choobi Bridge,

1151863411 Tehran, Iran

e-mail: a_hafez@azad.ac.ir; Hafezalkotob@iust.ac.ir

E. Haji-Sami

e-mail: paieez_sami@yahoo.com
}

\section{H. Omrani}

Department of Industrial Engineering, Urmia University

of Technology, Band road, 57155-419 Urmia, Iran

e-mail: omrani57@iust.ac.ir; h.omrani@uut.ac.ir
Keywords Data envelopment analysis - Discrete uncertain data $\cdot$ RDEA $\cdot$ Robust optimization

\section{Introduction}

In the highly competitive and dynamic markets derived from globalization, the domestic firms should find a competitive edge that enables them to survive in the market. Moreover, limited natural resources and growing environmental concerns and regulations about production processes are new considerations influence the firms' operations. Therefore, the operational efficiencies would play an important role in survival and growth of firms. Especially in electricity distribution companies, operational efficiency is the most crucial issue among regulators (Sadjadi and Omrani 2008).

Data envelopment analysis (DEA) is a well-known nonparametric technique that measures the relative operational efficiency of similar decision-making units (DMUs). The most important capability of DEA is its ability to compare several parameters (inputs/outputs) concurrently and sum up them into a scalar measure of relative efficiency. The efficiencies of DMUs are obtained from weights corresponding to each input and output that computed through the optimal solution of linear programming (LP) problems. In fact, DEA is a data-oriented method for measuring and benchmarking the relative efficiency of peer DMUs. Target setting and improvement of DMU's performance are important features of DEA technique. There are several successful real-world applications of DEA method in different public and private sector industries such as banks, software development, health care, pharmacies, auto manufacturing, fisheries and search engines (Saranga and Phani 2009). Sadjadi and Omrani (2008), for instance, used 
DEA method for measuring the relative efficiency of energy companies in Iran. Roghanian and Foroughi (2010) implemented DEA to compare efficiencies of all regional and international airports in Iran using different input/ output data. Goto and Tsutsui (1998) employed DEA approach to measure overall cost and technical efficiencies between Japanese and US electricity power plants. Saranga and Phani (2009) employed non-parametric DEA models and parametric methods such as regression analysis to specify the factors that have contributed to the internal operational efficiencies of firms in Indian pharmaceutical industry.

One of the most important issues associated with DEA is the uncertainty associated with the data. Since the resulted formulation of DEA technique is in form of LP, one can use traditional sensitivity analysis when there are one or a few uncertain parameters. However, when all input data are subject to uncertain, it is practically impossible to use sensitivity analysis method to handle all uncertainties. There are several methods for estimating the efficiencies of DMUs under data uncertainty.

In the real-world problems, data are often contaminated by perturbations (Izadi and Kimiagari 2014; Khalaj et al. 2013; Hosseini and Tarokh 2011; Shad et al. 2014). Because of perturbations in data, the efficient frontier in DEA is changed and the determined targets may become incorrect. Thus, the correction in the proposed target setting models would be necessary such that perturbation in inputs and outputs data would be considered (Monfared and Safi 2013; Bashiri et al. 2013). In a survey study, Ben-Tal and Nemirovski (2000) showed that a small perturbation on data could lead to infeasible solutions for some benchmark optimization problems. On the other hand, Bertsimas and Sim 2003, 2004; Bertsimas and Thiele 2006 and Bertsimas et al. (2004) developed new LP to adjust the robustness of the model against conservatism of the solution. In our LP reformation of DEA model, the results of the efficiency estimation and target setting could be unreliable in many cases especially when the efficiency of a particular firm is close to another. Mulvey et al. (1995) suggested an alternative approach, which is called scenario-based robust optimization (RO). This approach integrates goal programming formulations with a scenario-based description of problem data. It is a series of solutions of the model data from a scenario set. This motivates us to use robust DEA model to achieve more reliable results.

Sadjadi and Omrani (2008) developed a DEA model based on robust optimization approach and proposed a new formulation of DEA which is more reliable for efficiency estimating and ranking strategies. Also, they showed that the robust DEA founded upon Bertsimas and Sim 2003, 2004; Bertsimas and Thiele 2006) and
Bertsimas et al. (2004) is easier and more applicable than robust DEA based on Ben-Tal and Nemirovski (2000) approach. Robust optimization generally refers to the modeling of optimization problems with uncertain data to obtain a solution that is guaranteed to be good and feasible for all or most possible uncertain parameters Bashiri and Moslemi (2013). Uncertainty in the parameters is containing all (or most) possible values that may be realized for the corresponding parameters. Shokouhi et al. (2010) proposed DEA under uncertainty which was based on a robust optimization model that input and output parameters were constrained to be within an uncertainty set. They applied Monte Carlo simulation to compute the conformity of the ranking in the RDEA model.

Morita (2003) developed a method using DEA which dealt with the use of non-parametric production frontiers and did not require cost information on inputs and outputs for identifying the economies of scope. The most robust multipliers have been defined for evaluation of the dominance relation of efficient frontiers. Foroughi and Aouni (2012) determined efficiency based on DEA with interval data and setting up a full ranking of DMUs in two phases. At first, interval efficiencies have been computed; afterwards, they combined the lower and upper bounds of the interval efficiencies. Hatami-Marbini et al. (2012) developed a fuzzy DEA framework with a Banker, Charnes and Cooper (BCC) model for measuring crisp and interval efficiencies using alfa-level approach to convert $\mathrm{BCC}$ model into an interval programming model.

Our paper is closely related to Sadjadi and Omrani (2008). They studied robust DEA model under continuous uncertain data. To the best of authors' knowledge, no research was found that considers the discrete uncertainty regarding input and output data of DEA. Therefore, there are two main contributions in this study. For the first and foremost, we extend DEA model to scenario-based description of the uncertain data. Using RO approach of Mulvey et al. (1995), we develop DEA formulation to consider discrete uncertainty in input and output parameters as a set of possible scenarios. In the second place, we explore the effect of the discrete uncertain data on the degree of operational efficiency achieved by the Iranian electricity distribution companies (Satapathy and Mishra 2013).

The rest of the article is organized as follows. In "Data envelopment analysis (DEA)", the background of DEA approach has been described. In "Robust optimization", scenario-based robust approach based on Mulvey robust optimization has been expressed briefly. In "Robust DEA based on Mulvey approach", we formulate robust DEA model based on Mulvey approach. A real numerical example demonstrates the efficacy of the model in Iranian 
Electricity Company in "Case study". At the end, concluding remarks and some directions for future research are given in "Conclusion".

\section{Data envelopment analysis (DEA)}

DEA is a non-parametric approach which determines a piecewise linear efficiency frontier along the most efficient companies (DMUs) and derives the relative efficiency measures for all other companies (DMUs). The method was first introduced by Charnes et al. (1978) and has been widely implemented by many researchers in various sectors. DEA identifies an efficient frontier made up of the best practice DMUs to measure the relative efficiency scores of the less efficient DMUs. We choose an inputoriented approach of DEA to adjust the output by changing the input parameters such that the efficiency is maximized.

Assume that $n$ DMUs should be evaluated by DEA method where each DMU has $m$ input and $t$ output data. Let $x_{i j}$ denotes $i$ th input and $y_{t j}$ represents $t$ th output of DMU $j$. Moreover, let $u_{r}$ and $v_{i}$ be the dual variables associated with $x_{i j}$ and $y_{t j}$, respectively. The fractional DEA model is formulated as follows:

$\max z=\frac{\sum_{r=1}^{t} u_{r} y_{r 0}}{\sum_{i=1}^{m} v_{i} x_{i 0}}$,

s.t.

$\frac{\sum_{r=1}^{t} u_{r} y_{r j}}{\sum_{i=1}^{m} v_{i} x_{i j}} \leq 1, \quad j=1, \ldots, n$,

$u_{r}, v_{i} \geq 0$.

Model (1-3) is a non-linear programming problem (NLP). The DEA model is solved $n$ times to determine the relative efficiencies of different DMUs. Since model (1-4) is an NLP problem, Charnes et al. (1983) recommended a simple modification of the objective function to linearize the problem as follows:

$\max z=\sum_{r=1}^{t} u_{r 0} y_{r 0}$,

s.t.

$\sum_{r=1}^{t} u_{r} y_{r j}-\sum_{i=1}^{m} v_{i} x_{i j} \leq 0$,

$\sum_{i=1}^{m} v_{i} x_{i}=1$,

$u_{r}, v_{i} \geq 0, \quad \forall j=1,2, \ldots, n$.

LP problem (3-5) has been widely used for the past three decades and the results have been commonly accepted as measure of relative efficiencies of different DMUs. However, when there is uncertainty with regard to the inputs and the outputs data, specific techniques should be used to make sure that small changes in input/output data do not alter the resulted rankings.

\section{Robust optimization}

Classical modeling approaches in operation research under uncertainty assume full probabilistic characterizations. The learning which is needed to implement the policies derived from these models is accomplished either through classical statistical estimation procedures or subjective Bayesian priors. However, in many models, the uncertainty is ignored altogether, and a representative nominal value of the data is used simply (e.g., expected values). The classical approach to deal with uncertainty is stochastic programming (SP). Recently, RO is introduced as a complementary alternative to sensitivity analysis and SP. Indeed, RO, while not without limitations, has some pros over stochastic LP and it is more generally applicable. Soyster (1973) proposed the highest protection model of the nominal linear optimization problem which is the most conservative in practice in the sense of the robust solution. Ben-Tal and Nemirovski (2000) assumed that the true values of uncertain data entries in $i$ th inequality constraint are obtained from the nominal values of the entries by random perturbations.

The need for robustness has been recognized in a number of application areas. Mulvey et al. (1995) dealt with optimization problems that have two distinct components: a structural component that is fixed and free of any noise in its input data, and a control component that is subjected to noise in its input data. Then, they introduced two sets of variables to formulate such problems:

$x \in R^{n_{1}}$, represents the vector of decision variables that their optimal values are not dependent upon the realization of the uncertain parameters. They are also called design variables that cannot be adjusted once a specific realization of the data is observed.

$y \in R^{n_{2}}$, represents the vector of control decision variables that their optimal value are contingent upon the realization of uncertain parameters as well as the optimal value of the design variables.

Assume an LP model with the following structure:

$\min c^{T} x+d^{T} y, x \in R^{n_{1}}, y \in R^{n_{2}}$,

s.t.

$A x=b$,

$B x+C y=e$,

$x, y \geq 0$. 
The objective function (11) consists of design and control decision variables. Equation (12) represents the structural constraints that their coefficients are assumed fixed and free of noise. Equation (13) represents the control constraints that their coefficients and parameters are subject to noise.

To formulate the RO problem, Mulvey et al. (1995) defined a set of probable scenarios $\Omega=\{1,2, \ldots, S\}$ for LP model (14-15). To this end, for each scenario $s \in \Omega$, the set $\left\{d_{s}, B_{s}, C_{s}, e_{s}\right\}$ of realizations for the coefficients and parameters are associated. Moreover, the probability of scenarios are indicated by $p_{s}$, where $\left(\sum_{s=1}^{S} p_{s}=1\right)$ The optimal solution of problem (6-8) will be robust with respect to optimality if it stays "close" to optimal for any probable scenario $s \in \Omega$. It is then called solution robust. The solution is also robust with respect to feasibility if it stays "almost" feasible for any probable scenario $s \in \Omega$. It is then called model robust.

It is improbable that any solution to program (6-8) will stay both feasible and optimal for all scenarios indicated by $s \in \Omega$. If the system that is being modeled inherently has substantial redundancies built in, then it might be possible to achieve solutions that stay both feasible and optimal. Otherwise, the RO model proposed by Mulvey et al. (1995) enables us to measure the tradeoff between solution and model robustness. Let us define a set $\left\{y_{1}, y_{2}, \ldots, y_{s}\right\}$ of control variables for each scenario $s \in \Omega$. Additionally, let a set $\left\{z_{1}, z_{2}, \ldots, z_{s}\right\}$ be the error vectors that measure the infeasibility allowed in the control constraints under scenario $s \in \Omega$. Now, consider the formulation of the RO model as follows:

$\min \sigma\left(x, y_{1}, \ldots, y_{s}\right)+\omega \rho\left(z_{1}, \ldots, z_{s}\right)$,

s.t.

$A x=b$,

$B_{s} x+C_{s}+Z_{s}=e_{s}, \quad \forall s \in \Omega$,

$x_{s} \geq 0, y_{s} \geq 0, \quad \forall s \in \Omega$.

With multiple probable scenarios, the objective function $\xi=c^{T} x+d^{T} y$ turns into a random variable that takes the value $\xi_{s}=c^{T} x+d_{s}^{T} y_{s}$, with probability $p_{s}$. Therefore, there is no longer a unique choice for an aggregate objective. Term $\rho\left(z_{1}, z_{2}, \ldots, z_{s}\right)$ penalizes violations of the control under some of the scenarios. Different alternatives can be employed for penalty function and it is also problem dependent (Mulvey et al. 1995).For instance, $\rho\left(z_{1}, z_{2}, \ldots, z_{s}\right)=\sum_{s \in \Omega}\left(p_{s} z_{s}^{T} z_{s}\right)$ is a quadratic penalty function for equality constrained problems where both positive and negative violations should be penalized. Penalty function $\rho\left(z_{1}, z_{2}, \ldots, z_{s}\right)=\sum_{s \in \Omega} p_{s} \max \left\{0, z_{\mathrm{s}}\right\}$ can be applied for inequality control constraints when only positive violations should be penalized (i.e., negative values of show slack in the inequality constraints which are acceptable).By adjustment the goal programming weight $\omega$, the RO model is able to generate a spectrum of solutions that measure tradeoff between solution and model robustness.

Von Neumann and Morgenstern (2007) interpreted the risk as the variance of output. High variance of $\xi_{s}=$ $c^{T} x+d_{s}^{T} y_{s}$ shows that there is high fluctuation in outcome. Bar-Shira and Finkelshtain (1999) stated that using the function, which simultaneously raises the mean and reduces variance, is more robust than approaches based on expected value. The following equation demonstrates the mean-variance function for each scenario.

$\sigma\left(x, y_{1}, \ldots, y_{s}\right)=\sum_{s \in S} p_{s} \xi_{s}+\lambda \sum_{s \in S} p_{s}\left(\xi_{s}-\sum_{s^{\prime} \in S} p_{s}^{\prime} \xi_{s^{\prime}}\right)^{2}$.

An efficient frontier can be generated simply by parameterizing the tradeoff between risk and expected outcome (i.e., by changing $\lambda$, systematically).This formulation needs that the distribution of the random variable $\xi_{s}$ be symmetric around its means as well as the third and higher moments of $\xi_{s}$ are not considered (Mulvey et al. 1995).

\section{Robust DEA based on Mulvey approach}

As mentioned, it is almost impossible for DEA applications in many real cases to determine and capture the completely accurate data of the inputs and outputs. In other words, the real data are uncertain and the applications of the exact models could lead to incorrect results.

Since the DEA model (3-20) is an LP, uncertainty in output and input data (i.e., $x_{i j}$ and $y_{r j}$, respectively) can be formulated by RO model based on approach of Mulvey et al. (1995) [i.e., RO model (21-22)]. A set of scenario of probable input and output data is indicated by $\Omega=$ $\{1,2, \ldots, S\}$ with incidence probability $p_{s}$, for each scenario $s \in \Omega$.Therefore, the robust DEA model based on Mulvey approach is as follows:

$$
\begin{gathered}
\max \sum_{s \in \Omega} \sum_{r=1}^{t} p_{s} u_{r} y_{\text {ros }}-\gamma \sum_{s \in \Omega} p_{s} \delta_{s o} \\
-\lambda \sum_{s \in \Omega} p_{s}\left(\xi_{s}-\sum_{s^{\prime} \in \Omega} p_{s^{\prime}} \xi_{s^{\prime}}\right)^{2}
\end{gathered}
$$

s.t.

$\sum_{i=1}^{m} v_{i} x_{i o}+\delta_{s o}=1, \quad \forall s \in \Omega$, 
$\sum_{r=1}^{t} u_{r} y_{r j}-\sum_{i=1}^{m} v_{i} x_{i j}+\delta_{s j}=0, \quad \forall s \in \Omega, j=1, \ldots, n$,

$v_{i}, u_{r}, \delta_{s j} \geq 0, \quad \forall i, r, s$.

The objective function of the robust formulation of DEA has three terms. The first term is the expected efficiency of the DMUs. The second term is the variance of the efficiency weighted by the goal programming parameter $\gamma . \delta_{s j}$ is error variable under scenario $s$ for $\mathrm{DMU}_{j}$ which adjusts how much $\mathrm{DMU}_{j}$ can go out of feasibility space under $s$ scenario. The infeasibility penalty value is measured by term $\left(\sum_{s \in \Omega} p_{s} \delta_{s o}\right)$. Therefore, the third term of objective function (26) penalizes a norm of the infeasibilities, weighted by parameter $\lambda$. The coefficients $\gamma$ and $\lambda$ are userdefined parameters which identify the importance of variance and infeasibility terms, respectively. The robust formulation (13-15) is an NLP program and the problem can be more readily solved if it is transformed into an LP problem. Therefore, we use transformation variables $Q_{s}^{+}$ and $Q_{s}^{-}$for the quadratic term of the variance in the objective function. Therefore, NLP model (13-15) converts into the following LP model:

$$
\max \sum_{s \in \Omega} \sum_{r=1}^{t} p_{s} u_{r} y_{r o s}-\gamma \sum_{s \in \Omega} p_{s} \delta_{s o}-\lambda \sum_{s \in \Omega} p_{s}\left(Q_{s}^{+}+Q_{s}^{-}\right),
$$

s.t.

$\sum_{i=1}^{m} v_{i} x_{i o}+\delta_{s o}=1, \quad \forall s \in \Omega$,

$\sum_{r=1}^{t} u_{r} y_{r j}-\sum_{i=1}^{m} v_{i} x_{i j}+\delta_{s j}=0, \quad \forall s \in \Omega, j=1, \ldots, n$,

$$
\sum_{r=1}^{t} p_{s} u_{r} y_{r o s}-p_{s}\left(\sum_{s^{\prime} \in \Omega} \sum_{r=1}^{t} p_{s^{\prime}} u_{r} y_{r o s}\right)=Q_{s}^{+}-Q_{s}^{-},
$$$$
\forall s \in \Omega,
$$

$v_{i}, u_{r}, \delta_{s j}, Q_{s}^{+}, Q_{s}^{-} \geq 0, \quad \forall i, r, s$.

The variance term in objective function (13) is quadratic and it has been transformed into linear form using variables $Q_{s}^{+}$and $Q_{s}^{-}$. Constraint (19) computes the variance term of efficiency under each scenario. Term $\left(\sum_{s^{\prime} \in \Omega} \sum_{r=1}^{t} p_{s^{\prime}} u_{r} y_{r o s}\right)$ in constraint (19) is the expected value of efficiencies which indicates the amount of DMU's efficiency under probable scenarios. Since one of the variables $Q_{s}^{+}$and $Q_{s}^{-}$takes the positive value, this constraint measures the expected deviation of efficiency from its expected value such as variance. Therefore, the variance value in model (16-19) is demonstrated via term $\left(\sum_{s \in \Omega} p_{s}\left(Q_{s}^{+}+Q_{s}^{-}\right)\right)$in objective function.

\section{Case study}

To discuss the performance of the proposed robust DEA based on Mulvey approach, we employ the proposed method in the real problem of electricity distribution units. Since, Mulvey robust approach yields an NLP program, we implement the transformation variation to convert it into an LP problem [see model (16-19)].

The actual data of year 2008 for Iranian electricity distribution units (as DMUs) have been considered. The Iranian electricity distribution units, established in 1992, are public and operate under the supervision of TAVANIR Company $^{1}$ (Iran Power Generation, Transmission, and Distribution Management Company). TAVANIR has conceived that electricity distribution companies have high incentive to not report real and clear data. It may be beneficial for them to conceal real information and reveal deceptive input and output data. Moreover, real and accurate data about key performance criteria of all companies do not always exist. Therefore, it is important to TAVANIR to analyze efficiency of these companies under a large amount of uncertainty. The analyzers of TAVANIR are able to determine pessimistic, medium, and optimistic scenarios for output and input data of the companies. We propose RDEA to deal with these uncertain situations.

Jamasb and Pollitt (2000) extensively reviewed the electricity international case studies and identified the most often used inputs as operating cost, the number of employees, transformer capacity and network length. Moreover, the most frequently used outputs are recognized as units of energy delivered, the number of customers and the size of service area, as well. Hence, similar to Sadjadi and Omrani (2008), we take account of five parameters as inputs and output data for estimating the operational efficiency of the electricity distribution units. The inputs are the number of labors, transformer capacity, and network length. The outputs are also units of total electricity sales and the number of customers. It is noteworthy that the measurement units for transformer capacity, the network length and total electricity sales are MVA, Kilometer $(\mathrm{Km})$ and MWh, respectively.

1 TAVANIR is responsible for electricity generation, transmission, and distribution in Iran. This company operates under the supervision of Ministry of Energy. 
The case study contains annual data on 38 companies observed in 2008 obtained from Power Generation, Transmission, and Distribution Management Company publications. Note that in the real world, these data are not precise and they are estimated with a specific error level. For instance, the network length and transformers capacity data for electricity distribution units are capital parameters and their actual values are not often available. Moreover, total electricity sales and number of customers are not often reported precisely. We take three scenarios into account for data that are called pessimistic, medium, and optimistic scenarios. The occurrence probabilities of these scenarios are estimated as $0.25,0.5$, and 0.25 , respectively. Table 1 demonstrates the inputs and outputs of units under these scenarios.

( $\mathrm{s}_{1}=$ Pessimistic, $\mathrm{s}_{2}=$ Medium, $\mathrm{s}_{3}=$ Optimistic $)$.

The robust DEA model based on Mulvey approach has been applied to evaluate 38 companies for Iranian electricity distribution units under imprecise data in year 2008. In this model, the expected value and variance impact of probable scenarios have been considered. As mentioned earlier, tradeoff between solution robustness and model robustness can be derived by penalty parameter $\gamma$. Figure 1 illustrates the effects of the penalty function on the expected value of efficiency of DMUs. We know from the figure that the expected value of efficiency of DMUs is decreasing with the penalty parameter $\gamma$. On the other hand, by parameterizing the value $\lambda$, the tradeoff between expected value and variance can be constructed. The larger the parameter $\lambda$, the more importance of variance of scenarios will be. Figure 2 demonstrates the effects of parameter $\lambda$ on efficiency of DMUs, as well. From the figure, we know that when $\lambda$ increases, the expected value of efficiency decreases. From these figures, it is also found that in these cases, the parameters $\gamma$ and $\lambda$ significantly alter DMUs' efficiencies, but they rarely change DMUs ranking. Note that in other cases, when there are considerable variations among probable scenarios, changing the parameter $\lambda$ may remarkably alters the ranking as well.

To get deep insight from robust DEA model (16-19), we compare all terms of objective function systematically. To this end, the goal programming parameters $\gamma$ and $\lambda$ are assumed fixed $(\gamma=3 \& \lambda=0.8)$. Solving the robust DEA model, all terms of objective function are calculated and reported in columns of Table 2. Next to each column, the ranking is indicated as well.

Now, let us consider the probable scenarios in more detail. The pessimistic efficiency value of each DMU is the worst and the optimistic efficiency of each DMU is the best value of efficiency amount, which are found by efficiency ranking under probable scenarios. For calculating the efficiencies under different scenarios, we should set appropriate values for probabilities. For instance, under pessimistic scenario, we should set $p_{1}=1, p_{2}=0$, and $p_{3}=0$. The obtained efficiencies for DMUs in pessimistic, medium, and optimistic scenarios are indicated in Table 2. The efficiencies in these cases also give us the ranking of DMUs in standard DEA formulation under each probable scenario. Comparing the ranking of DMUs, we found that the position of the electricity distribution units has slightly changed under different probable scenarios.

As we saw earlier, different efficiencies and rankings have obtained under various scenarios. Robust DEA model (16-19) gives us an aggregate measure. Expected value and variance terms of the model are indicated in Table 2. In the last column of the table, expected value and variance measures are integrated with coefficient $\lambda$. The penalty function $\sum_{s \in \Omega} p_{s} \delta_{s o}$ estimates the infeasibility allowed in different scenarios. The infeasibility penalty measure associated with each DMU is also reported in the table. The highest rank of infeasibility penalty measure demonstrates the electricity distribution unit which has the lowest infeasibility measure.

From the table we found that, the 20th electricity distribution unit is the highest efficient unit. For this DMU, the efficiencies under pessimistic, medium, and optimistic scenarios are 0.81981982, 0.9009009, and 1, respectively. Hence, the expected value of the efficiency of this unit would be 0.90540541 . According to the infeasibility penalty measures, we derive that 20th DMU has a low influence on the quality of its efficiency. Total objective value of robust DEA for this DMU is 0.6658089; therefore, it attains the first rank. Moreover, we obtain that 22th DMU has a high influence on the quality of its efficiency where the total objective value of robust DEA for this DMU is 0.1558; hence, it means that 22th DMU attains the last position in all DMUs ranking. Moreover, we derive from the Table 2, that the changing in parameters $\lambda$ and $\gamma$ does not trigger a dramatic change in ranking of DMUs.

\section{Conclusion}

Uncertainty is an inherent part of the real performance evaluation problems. On the one hand, some precise real data about companies may not always available, on the other hand, some companies may have an incentive to conceal their real output and input data. Although, two approaches of robust optimization for DEA was proposed by Sadjadi and Omrani (2008), discrete data uncertainty based on probable scenarios has not been considered yet. For scenario-based uncertainty about input and output data, we presented a new robust DEA model founded upon the approach of Mulvey et al. (1995). One of the main advantages of this approach is that it enables decision makers to draw a tradeoff between 


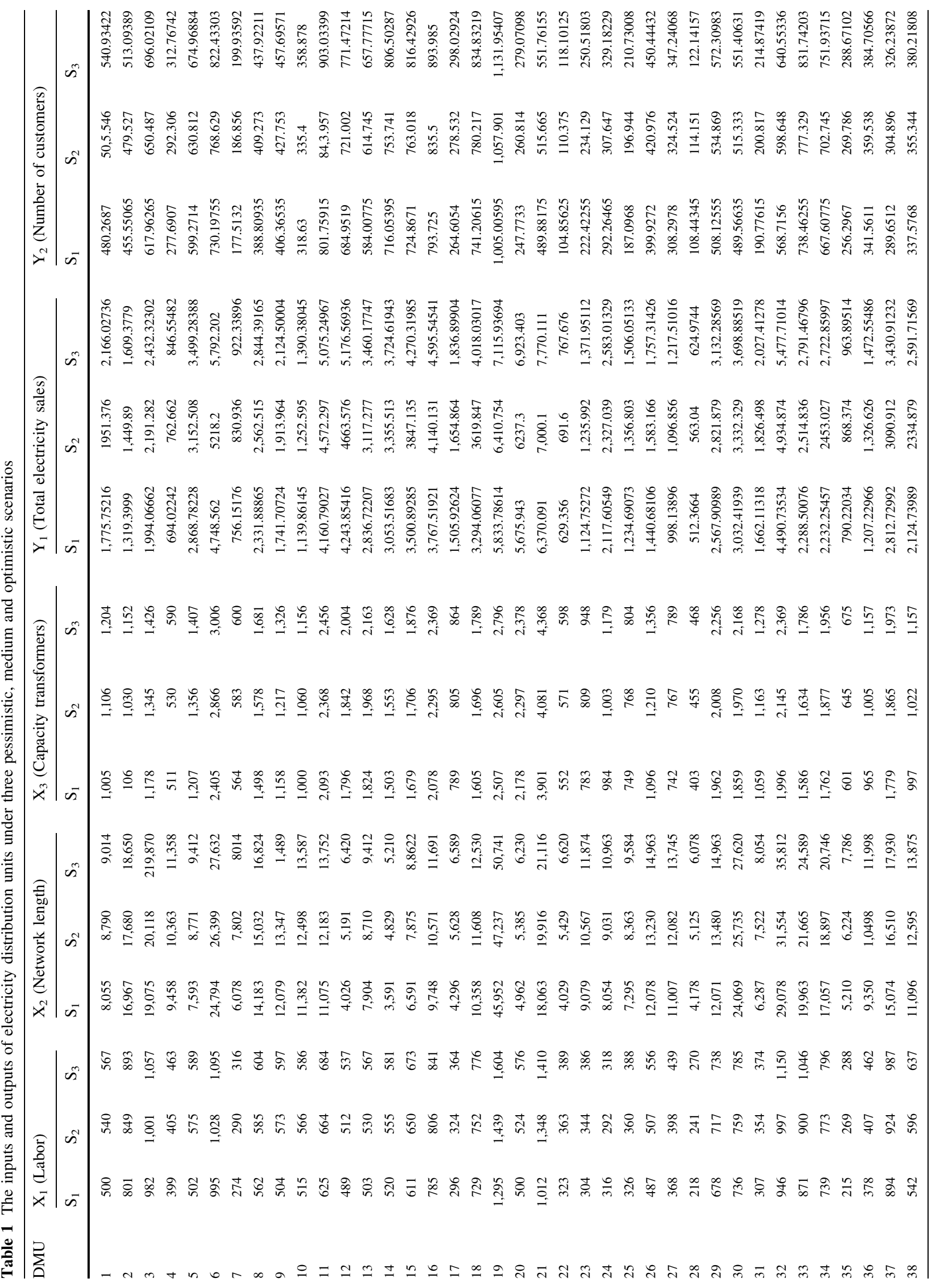




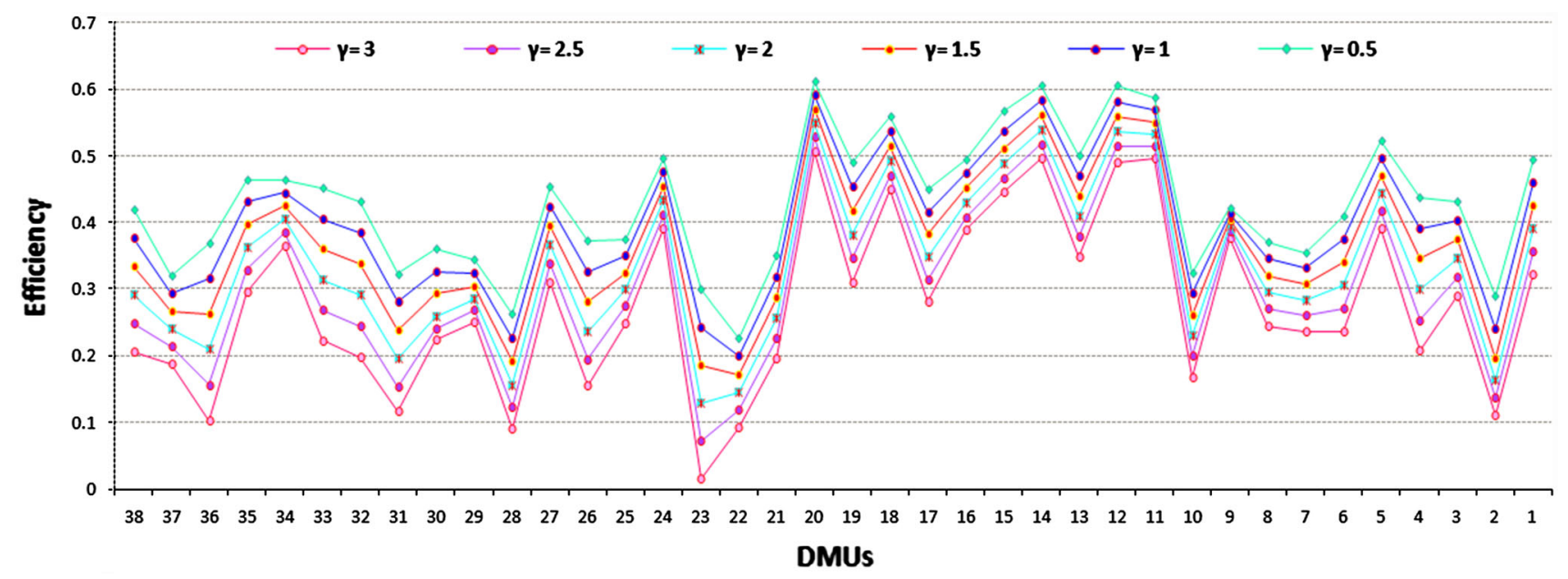

Fig. 1 Comparison of objective function under $\lambda=0.8$ and different values of $\gamma$

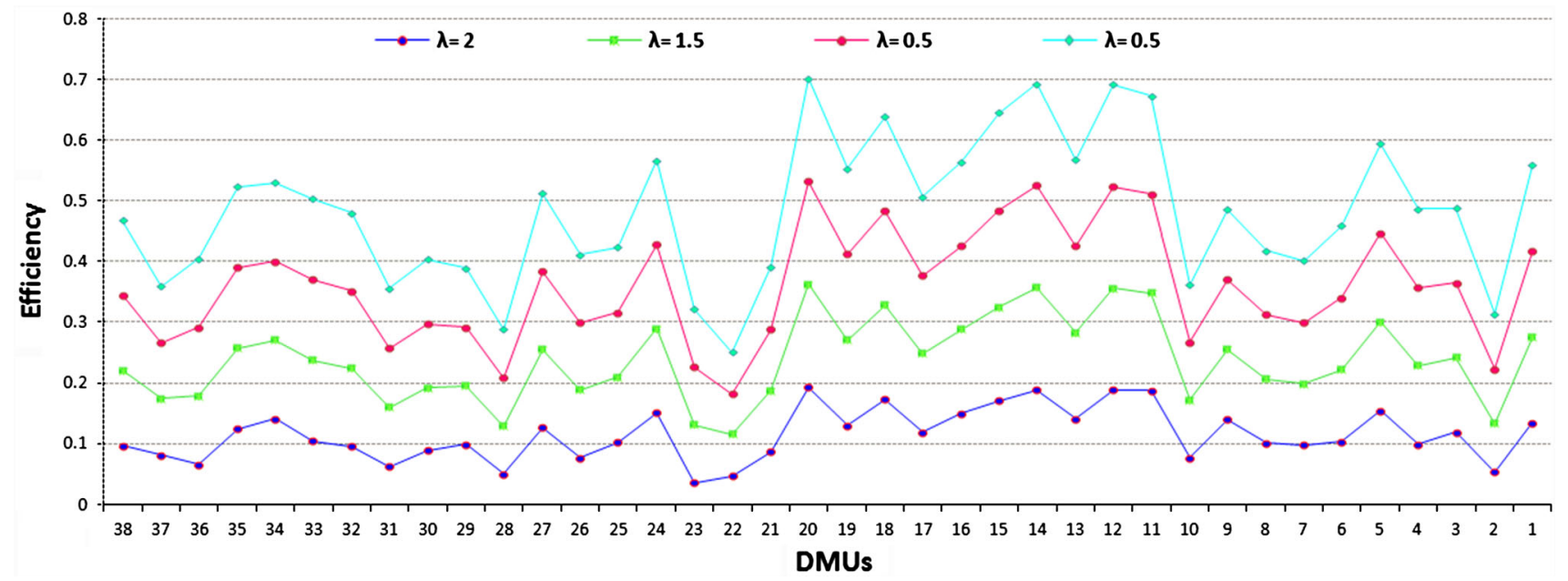

Fig. 2 Comparison of objective function under $\gamma=0.8$ and different values of $\lambda$

expected value and variance of DMUs' efficiency under probable scenarios, and a tradeoff between solution and model robustness. We implemented the results of the proposed model using data gathered from an Iranian energy organization when optimistic, medium, and pessimistic scenarios exist about their reported data. Our preliminary results indicate that our robust DEA approach can provide analysts with more decision criteria under uncertain condition. Moreover, sensitivity analyses on parameters $\lambda$ and $\gamma$ can derive a spectrum of solutions that may be useful for managerial tradeoffs.

Although our model is restricted to input-oriented model and constant return to scale, one can easily generalize it to output-oriented model and variable return to scale. Our model considers statics situation, however, it can be readily developed to dynamic situation to adapt the model in multi-period real problems. There are also other directions and suggestions for future research. First, we assumed that all outputs are desirable; however, the real problem may be the undesirable output data, decreasing the amount of which is favorable. Extending our robust DEA model for both desirable and undesirable data is interesting. Second, the robust DEA model can be developed into the two-stage or network DEA models where output of a DMU becomes input data of other DMU(s). Eventually, the parameters of the proposed model may be changed during the planning horizon. In this situation, we can expand the suggested model into the Malmquist model in dynamic condition. Since the return to scale models is linear, if the discrete uncertainties have been observed in data, one can utilize our approach to analyze the efficiency of DMUs. It means that all four models of CCR, BCC, CCR-BCC, and BCC- 
Table 2 All terms of objective function of the Robust DEA model under $\lambda=0.8$ and $\gamma=3$

\begin{tabular}{|c|c|c|c|c|c|c|c|c|c|c|c|c|c|c|c|c|}
\hline \multirow[t]{2}{*}{ DMU } & \multicolumn{2}{|c|}{$\begin{array}{l}\text { Pessimistic } \\
\text { efficiency }\end{array}$} & \multicolumn{2}{|c|}{$\begin{array}{l}\text { Medium } \\
\text { efficiency }\end{array}$} & \multicolumn{2}{|c|}{$\begin{array}{l}\text { Optimistic } \\
\text { efficiency }\end{array}$} & \multicolumn{2}{|c|}{$\begin{array}{l}\text { Expected } \\
\text { value of } \\
\text { efficiency }\end{array}$} & \multicolumn{2}{|c|}{$\begin{array}{l}\text { Variance } \\
\text { measure }\end{array}$} & \multicolumn{2}{|c|}{$\begin{array}{l}\text { Infeasibility } \\
\text { penalty } \\
\text { measure }\end{array}$} & \multicolumn{2}{|c|}{$\begin{array}{l}\text { Objective } \\
\text { function }\end{array}$} & \multicolumn{2}{|c|}{$\begin{array}{l}\text { Expected } \\
\text { value- } \lambda \\
*(\text { variance })\end{array}$} \\
\hline & Value & Rank & Value & Rank & Value & Rank & Value & Rank & Value & Rank & Value & Rank & Value & Rank & Value & Ranl \\
\hline 1 & 0.7137 & 8 & 0.7512 & 9 & 0.8038 & 11 & 0.7550 & 9 & 0.2827 & 9 & 0.0686 & 11 & 0.4556 & 13 & 0.5289 & 9 \\
\hline 2 & 0.3551 & 37 & 0.3738 & 37 & 0.3999 & 37 & 0.3756 & 37 & 0.1406 & 37 & 0.0504 & 23 & 0.1778 & 35 & 0.2631 & 37 \\
\hline 3 & 0.6205 & 20 & 0.6532 & 21 & 0.6989 & 21 & 0.6564 & 21 & 0.2458 & 21 & 0.0564 & 19 & 0.4058 & 17 & 0.4598 & 21 \\
\hline 4 & 0.6525 & 16 & 0.6868 & 17 & 0.7349 & 18 & 0.6902 & 17 & 0.2584 & 17 & 0.0917 & 4 & 0.3294 & 25 & 0.4835 & 17 \\
\hline 5 & 0.7133 & 9 & 0.7794 & 7 & 0.8610 & 7 & 0.7833 & 7 & 0.2932 & 7 & 0.0526 & 22 & 0.5282 & 7 & 0.5487 & 7 \\
\hline 6 & 0.5773 & 22 & 0.6296 & 22 & 0.6945 & 22 & 0.6328 & 22 & 0.2369 & 22 & 0.0687 & 10 & 0.3482 & 21 & 0.4432 & 22 \\
\hline 7 & 0.5029 & 28 & 0.5385 & 28 & 0.5848 & 29 & 0.5412 & 28 & 0.2026 & 28 & 0.0474 & 26 & 0.3317 & 24 & 0.3791 & 28 \\
\hline 8 & 0.5230 & 26 & 0.5621 & 26 & 0.6123 & 26 & 0.5649 & 26 & 0.2115 & 26 & 0.0500 & 24 & 0.3446 & 22 & 0.3957 & 26 \\
\hline 9 & 0.5713 & 23 & 0.6131 & 23 & 0.6671 & 23 & 0.6161 & 23 & 0.2307 & 23 & 0.0181 & 38 & 0.4854 & 11 & 0.4316 & 23 \\
\hline 10 & 0.4797 & 30 & 0.5049 & 31 & 0.5403 & 32 & 0.5074 & 31 & 0.1900 & 31 & 0.0623 & 15 & 0.2577 & 31 & 0.3555 & 31 \\
\hline 11 & 0.8159 & 4 & 0.8589 & 4 & 0.9190 & 4 & 0.8631 & 4 & 0.3231 & 4 & 0.0362 & 35 & 0.6475 & 4 & 0.6046 & 4 \\
\hline 12 & 0.8212 & 2 & 0.8924 & 2 & 0.9813 & 2 & 0.8968 & 2 & 0.3357 & 2 & 0.0456 & 27 & 0.6486 & 3 & 0.6282 & 2 \\
\hline 13 & 0.7170 & 7 & 0.7547 & 8 & 0.8075 & 10 & 0.7585 & 8 & 0.2840 & 8 & 0.0608 & 16 & 0.4818 & 12 & 0.5313 & 8 \\
\hline 14 & 0.8301 & 1 & 0.8923 & 3 & 0.9724 & 3 & 0.8968 & 3 & 0.3357 & 3 & 0.0441 & 29 & 0.6532 & 2 & 0.6282 & 3 \\
\hline 15 & 0.7763 & 5 & 0.8172 & 6 & 0.8744 & 6 & 0.8213 & 6 & 0.3075 & 6 & 0.0434 & 30 & 0.5891 & 6 & 0.5753 & 6 \\
\hline 16 & 0.6795 & 11 & 0.7153 & 12 & 0.7654 & 12 & 0.7189 & 12 & 0.2691 & 12 & 0.0384 & 34 & 0.5159 & 9 & 0.5036 & 12 \\
\hline 17 & 0.6305 & 18 & 0.6870 & 16 & 0.7573 & 14 & 0.6905 & 16 & 0.2585 & 16 & 0.0677 & 13 & 0.4017 & 18 & 0.4837 & 16 \\
\hline 18 & 0.7711 & 6 & 0.8260 & 5 & 0.8975 & 5 & 0.8302 & 5 & 0.3108 & 5 & 0.0441 & 28 & 0.5947 & 5 & 0.5815 & 5 \\
\hline 19 & 0.6876 & 10 & 0.7493 & 10 & 0.8260 & 8 & 0.7530 & 10 & 0.2819 & 10 & 0.0727 & 8 & 0.4414 & 14 & 0.5275 & 10 \\
\hline 20 & 0.8198 & 3 & 0.9009 & 1 & 1.0000 & 1 & 0.9054 & 1 & 0.3390 & 1 & 0.0424 & 32 & 0.6658 & 1 & 0.6342 & 1 \\
\hline 21 & 0.4910 & 29 & 0.5373 & 29 & 0.5943 & 27 & 0.5400 & 29 & 0.2022 & 29 & 0.0603 & 17 & 0.2920 & 29 & 0.3783 & 29 \\
\hline 22 & 0.3303 & 38 & 0.3601 & 38 & 0.3970 & 38 & 0.3619 & 38 & 0.1355 & 38 & 0.0537 & 20 & 0.1558 & 37 & 0.2535 & 38 \\
\hline 23 & 0.4712 & 32 & 0.5054 & 30 & 0.5498 & 30 & 0.5079 & 30 & 0.1902 & 30 & 0.1136 & 1 & 0.1040 & 38 & 0.3558 & 30 \\
\hline 24 & 0.6769 & 12 & 0.7359 & 11 & 0.8095 & 9 & 0.7395 & 11 & 0.2769 & 11 & 0.0425 & 31 & 0.5204 & 8 & 0.5180 & 11 \\
\hline 25 & 0.5206 & 27 & 0.5678 & 25 & 0.6264 & 25 & 0.5707 & 25 & 0.2136 & 25 & 0.0499 & 25 & 0.3501 & 19 & 0.3997 & 25 \\
\hline 26 & 0.5253 & 25 & 0.5530 & 27 & 0.5917 & 28 & 0.5558 & 27 & 0.2081 & 27 & 0.0776 & 7 & 0.2541 & 32 & 0.3893 & 27 \\
\hline 27 & 0.6504 & 17 & 0.6846 & 18 & 0.7326 & 19 & 0.6881 & 18 & 0.2576 & 18 & 0.0573 & 18 & 0.4306 & 15 & 0.4820 & 18 \\
\hline 28 & 0.3764 & 36 & 0.4096 & 36 & 0.4510 & 36 & 0.4116 & 36 & 0.1541 & 36 & 0.0658 & 14 & 0.1631 & 36 & 0.2883 & 36 \\
\hline 29 & 0.4793 & 31 & 0.5045 & 32 & 0.5398 & 33 & 0.5070 & 32 & 0.1898 & 32 & 0.0346 & 36 & 0.3404 & 23 & 0.3552 & 32 \\
\hline 30 & 0.4341 & 34 & 0.4570 & 35 & 0.4889 & 35 & 0.4592 & 35 & 0.1719 & 35 & 0.0322 & 37 & 0.3057 & 28 & 0.3217 & 35 \\
\hline 31 & 0.4323 & 35 & 0.4706 & 34 & 0.5183 & 34 & 0.4729 & 34 & 0.1770 & 34 & 0.0715 & 9 & 0.2030 & 34 & 0.3313 & 34 \\
\hline 32 & 0.6228 & 19 & 0.6800 & 19 & 0.7507 & 16 & 0.6834 & 19 & 0.2558 & 19 & 0.0936 & 3 & 0.3178 & 27 & 0.4787 & 19 \\
\hline 33 & 0.6710 & 13 & 0.7063 & 14 & 0.7557 & 15 & 0.7098 & 14 & 0.2657 & 14 & 0.0912 & 5 & 0.3482 & 20 & 0.4972 & 14 \\
\hline 34 & 0.6541 & 15 & 0.6885 & 15 & 0.7367 & 17 & 0.6919 & 15 & 0.2590 & 15 & 0.0398 & 33 & 0.4867 & 10 & 0.4847 & 15 \\
\hline 35 & 0.6688 & 14 & 0.7085 & 13 & 0.7625 & 13 & 0.7121 & 13 & 0.2666 & 13 & 0.0678 & 12 & 0.4203 & 16 & 0.4988 & 13 \\
\hline 36 & 0.5701 & 24 & 0.6001 & 24 & 0.6421 & 24 & 0.6031 & 24 & 0.2258 & 24 & 0.1063 & 2 & 0.2094 & 33 & 0.4225 & 24 \\
\hline 37 & 0.4510 & 33 & 0.4929 & 33 & 0.5447 & 31 & 0.4954 & 33 & 0.1855 & 33 & 0.0530 & 21 & 0.2750 & 30 & 0.3470 & 33 \\
\hline 38 & 0.6037 & 21 & 0.6582 & 20 & 0.7259 & 20 & 0.6615 & 20 & 0.2477 & 20 & 0.0856 & 6 & 0.3227 & 26 & 0.4634 & 20 \\
\hline
\end{tabular}

CCR can be appropriately altered by the robust optimization approach.

Open Access This article is distributed under the terms of the Creative Commons Attribution License which permits any use, distribution, and reproduction in any medium, provided the original author(s) and the source are credited.

\section{References}

Bar-Shira Z, Finkelshtain I (1999) Two-moments decision models and utility-representable preferences. J Econ Behave Organ 38:237-244. doi:10.1016/S0167-2681(99)00008-6

Bashiri M, Moslemi A (2013) The analysis of residuals variation and outliers to obtain robust response surface. J Ind Eng Int 9(1):1-10 
Bashiri M, Farshbaf-Geranmayeh A, Mogouie H (2013) A neuro-data envelopment analysis approach for optimization of uncorrelated multiple response problems with smaller the better type controllable factors. J Ind Eng Int 9(1):30

Ben-Tal A, Nemirovski A (2000) Robust solutions of linear programming problems contaminated with uncertain data. Math Program 88(3):411-424

Bertsimas D, Sim M (2003) Robust discrete optimization and network flows. Math Progr 98:49-71. doi:10.1007/s10107-003-0396-4

Bertsimas D, Sim M (2004) The price of robustness. Oper Res 52:35-53. doi:10.1287/opre.1030.0065

Bertsimas D, Thiele A (2006) A robust optimization approach to inventory theory. Oper Res 54:150-168. doi:10.1287/opre.1050. 0238

Bertsimas D, Pachamanova D, Sim M (2004) Robust linear optimization under general norms. Oper Res Lett 32:510-516. doi:10. 1016/j.orl.2003.12.007

Charnes A, Cooper WW, Rhodes E (1978) Measuring the efficiency of decision making units. Eur J Oper Res 2:429-444. doi:10. 1016/0377-2217(78)90138-8

Charnes A, Cooper WW, Seiford L, Stutz J (1983) Invariant multiplicative efficiency and piecewise Cobb-Douglas envelopments. Oper Res Lett 2:101-103. doi:10.1016/0167-6377(83)90014-7

Foroughi AA, Aouni B (2012) Ranking units in DEA based on efficiency intervals and decision-maker's preferences. Int Trans Oper Res 19:567-579. doi:10.1111/j.1475-3995.2011.00834.x

Goto M, Tsutsui M (1998) Comparison of productive and cost efficiencies among Japanese and US electric utilities. Omega-Int J Manage S26:177-194. doi:877a01e2e3e3d0aa067a8d136704a38b

Hatami-Marbini A, Tavana M, Saati S, Agrell PJ (2012) Positive and normative use of fuzzy DEA-BCC models: a critical view on NATO enlargement. Int T Oper Res 20:411-433. doi:10.1111/j. 1475-3995.2012.00871.x

Hosseini MB, Tarokh MJ (2011) Interval type-2 fuzzy set extension of DEMATEL method. In: Computational Intelligence and Information Technology, vol 250. Springer, Berlin, Heidelberg, pp 157-165

Izadi A, Kimiagari AM (2014) Distribution network design under demand uncertainty using genetic algorithm and Monte Carlo simulation approach: a case study in pharmaceutical industry. J Ind Eng Int 10(1):1
Jamasb T, Pollitt M (2000) Benchmarking and regulation: international electricity experience. Utilities Policy 9:107-130. doi:272cd35c9c5e488b5e93b23f124f38b8

Khalaj M, Khalaj F, Khalaj A (2013) A novel risk-based analysis for the production system under epistemic uncertainty. J Ind Eng Int 9(1):35

Monfared MAS, Safi M (2013) Network DEA: an application to analysis of academic performance. J Ind Eng Int 9(1):1-10

Morita H (2003) Analysis of economies of scope by data envelopment analysis: comparison of efficient frontiers. Int Trans Oper Res 10:393-402. doi:10.1111/1475-3995.00415

Mulvey JM, Vanderbei RJ, Zenios SA (1995) Robust optimization of large scale system. Operres 43:264-281. doi:10.1287/opre.43.2. 264

Roghanian E, Foroughi A (2010) An empirical study of Iranian regional airports using robust data envelopment analysis. Int $\mathbf{J}$ Ind Engine Comput 1:65-72. doi:10.5267/j.ijiec.2010.01.006

Sadjadi SJ, Omrani H (2008) Data envelopment analysis with uncertain data: an application for Iranian electricity distribution companies. Energy Policy 36:4247-4254. doi:10.1016/j.enpol. 2008.08.004

Saranga H, Phani BV (2009) Determinants of operational efficiencies in the Indian pharmaceutical industry. Int Trans Oper Res 16:109-130. doi:10.1111/j.1475-3995.2009.00668.x

Satapathy S, Mishra P (2013) A customer oriented systematic framework to extract business strategy in Indian electricity services. J Ind Eng Int 9(1):33

Shad Z, Roghanian E, Mojibian F (2014) Integration of QFD, AHP, and LPP methods in supplier development problems under uncertainty. J Ind Eng Int 10(1):1-9

Shokouhi AH, Hatami-Marbini A, Tavana M, Saati S (2010) A robust optimization approach for imprecise data envelopment analysis. Comput Ind Eng 59:387-397. doi:10.1016/j.cie.2010.05.011

Soyster AL (1973) Technical Note-convex programming with setinclusive constraints and applications to inexact linear programming. Oper res 21:1154-1157. http://www.jstor.org/stable/ 168933

Von Neumann J, Morgenstern O (2007) Theory of games and economic behavior (commemorative edition). Princeton university press, New Jersey 